\title{
Some $\mathrm{L}_{2}$ Markoff Inequalities
}

\author{
Lawrence F. Shampine ${ }^{1}$
}

\section{California Institute of Technology, Pasadena, Calif. and}

\section{Sandia Laboratory, Albuquerque, N. Mex.}

(June 15, 1965)

A number of authors have studied inequalities for polynomials of degree $n$ of the form

$$
\left\|\frac{d}{d x} P_{n}(x)\right\| \leqslant A n^{p}\left\|P_{n}(x)\right\| .
$$

\begin{abstract}
Iterated use of such inequalities give inequalities for higher derivatives. This paper determines the power $p$ and constant $A$ for the second derivatives for certain weighted $L_{2}$ norms. The iterated inequalities are not sharp.
\end{abstract}

Various authors have studied inequalities for polynomials of degree $n$ of the type

$$
\left\|\frac{d}{d x} P_{n}(x)\right\| \leqslant A n^{p}\left\|P_{n}(x)\right\| .
$$

When the norm is the maximum norm, A. Markoff has established the best possible constant $A$, power $p$, and the extremal polynomials. Repeated application of his inequality gives similar inequalities for higher derivatives which, however, are not sharp. W. Markoff has obtained best possible results for the higher derivatives.

E. Schmidt $[1]^{2}$ has obtained the power $p$ and asymptotic estimates of $A$ for the weighted $L_{2}$ norms giving rise to Legendre, Laguerre, and Hermite polynomials. The Hermite case is easy to solve for all derivatives essentially because the derivative of a Hermite polynomial is again a (multiple of a) Hermite polynomial. Best possible results are known in the Laguerre case for the first derivative but for no higher derivatives.

We shall look at the second derivatives in the Legendre and Laguerre cases. It turns out that as for the maximum norm the repeated use of the results for the first derivative are not sharp. The Laguerre case will be treated by a technique due to the author and the Legendre case by a development of Schmidt's technique. The first method is the simpler and is much more satisfactory as regards the extremal polynomials. The second method is capable of the sharper estimate of the constant.

${ }^{1}$ This work was supported in part by the National Science Foundation and in part by the United States Atomic Energy Commission. Reproduction in whole or in part is permitted for any purpose of the U.S. Government.

${ }^{2}$ Figures in brackets indicate the literature references at the end of this paper.
I. Here we look at

$$
\int_{0}^{\infty}\left[P^{\prime \prime}(x)\right]^{2} e^{-x} d x \leqslant M_{n}^{2} \int_{0}^{\infty}[P(x)]^{2} e^{-x} d x
$$

We use the Laguerre polynomials [2, p. 51]

$$
L_{j}(x)=\sum_{k=0}^{j}(-1)^{k} \frac{1}{k !}\left(\begin{array}{l}
j \\
k
\end{array}\right) x^{k} \quad j=0,1, \ldots
$$

for which

$$
\left(L_{i}, L_{j}\right)=\int_{0}^{\infty} L_{i}(x) L_{j}(x) e^{-x} d x=\delta_{i j}
$$

to express any polynomial $P(x)$ of degree $n$ as

$$
P(x)=\sum_{j=0}^{n} a_{j} L_{j}(x)
$$

With this basis

$$
\|P\|^{2}=(P, P)=\sum_{j=0}^{n} a_{j}^{2}, \quad\left\|P^{\prime \prime}\right\|^{2}=\sum_{i, j=0}^{n} a_{i} a_{j} b_{i j}
$$

where

$$
b_{i j}=\int_{0}^{\infty} L_{i}^{\prime \prime}(x) L_{j}^{\prime \prime}(x) e^{-x} d x .
$$


Two integrations by parts shows

$$
b_{i j}=L_{j}(0) L_{i}^{(3)}(0)-L_{j}(0) L_{i}^{\prime \prime}(0)-L_{j}^{\prime}(0) L_{i}^{\prime \prime}(0), i \leqslant j
$$

and from (1.2) we find

$$
b_{i j}=\frac{i(i-1)}{6}(3 j-i-1) \quad 0 \leqslant i \leqslant j \leqslant n .
$$

The solution of (1.1) is equivalent to finding $M_{n}^{2}$ for which

$$
M_{n}^{2}=\max \left\|P^{\prime \prime}\right\|^{2} \text { for }\|P\|^{2}=1
$$

From (1.3) we see that the maximum of this quadratic form is the largest eigenvalue of the (symmetric) matrix $B=\left(b_{i j}\right) i, j=2, \ldots, n$. We recall the results from the theory of positive matrices that the largest eigenvalue in modulus is real, positive, and simple. From (1.3) we see that $B$ is positive definite.

The idea of the technique to be used is as follows. $B$ is divided by an appropriate power of $n$. The resulting system of matrices is regarded as arising from the numerical solution of a fixed integral equation. The dominant eigenvalue and its eigenfunction of the integral equation represent limiting values for the system of matrices.

It is somewhat more convenient to change from the matrices $B$ to kernels. The eigenvalue problem for the kernel $K^{(n)}(x, y)$ on $0 \leqslant x, y \leqslant 1$ defined by

$$
K^{(n)}(x, y)=K_{i, j}^{(n)}\left\{\begin{array}{l}
\frac{i-1}{n}<x \leqslant \frac{i}{n} \\
\frac{j-1}{n}<y \leqslant \frac{j}{n}
\end{array} \quad i, j=1, \ldots ., n\right.
$$

is equivalent to that of the matrix $\frac{1}{n}\left(K_{i j}\right)$ with the definition $\varphi(x)=\varphi\left(\frac{i}{n}\right)=\varphi_{i}$ for $\frac{i-1}{n}<x \leqslant \frac{i}{n}$.

Define

$$
K_{i j}^{(n)}=\frac{1}{n^{3}} b_{i j}=\frac{1}{6}\left(\frac{i}{n}\right)\left(\frac{i}{n}-\frac{1}{n}\right)\left[3\left(\frac{j}{n}\right)-\left(\frac{i}{n}\right)-\frac{1}{n}\right] .
$$

It is clear the kernels converge (pointwise) to

$$
\tilde{K}(x, y)=\frac{x^{2}}{6}(3 y-x) \quad x \leqslant y .
$$

The eigenvalue problem $\lambda \varphi=\tilde{K} \varphi$ is equivalent to

$$
\begin{gathered}
\lambda \varphi^{(4)}(x)=\varphi(x) \\
\varphi(0)=\varphi^{\prime}(0)=0=\varphi^{\prime \prime}(1)=\varphi^{\prime \prime \prime}(1) .
\end{gathered}
$$

The solutions are [3, p. 525] for $k^{4}=\lambda^{-1}$

$(\cosh k+\cos k)(\sinh k x-\sin k x)$

$$
-(\sinh k+\sin k)(\cosh k x-\cos k x)
$$

and the $k$ are the solutions of

$$
1+\cos k \cosh k=0 .
$$

Plummer [4] has determined the smallest root $k_{0}$ to be 1.8751041 .

Let $\lambda, \lambda^{(n)}, M_{n}^{2}$ be the largest eigenvalues of $\tilde{K}, K^{(n)}$, $B$ respectively. A theorem of $\mathrm{W}$ eyl [5] gives the result

$$
\left|\tilde{\lambda}-\lambda^{(n)}\right| \leqslant\left\|\tilde{K}-K^{(n)}\right\| \leqslant \max \mid \tilde{K}(x, y)
$$

$$
-K^{(n)}(x, y) \mid=\frac{1}{2 n}-\frac{1}{6 n^{2}}
$$

Using the facts that $\tilde{K}>K^{(n)}>0$ implies the same inequality in the eigenvalues and that

$$
\lambda^{(n)}=\frac{1}{n^{4}} M_{n}^{2},
$$

we have the

Theorem. If $\mathrm{P}(\mathrm{x})$ is a polynomial of degree $\mathrm{n}$, then

$$
\int_{0}^{\infty}\left[\mathrm{P}^{\prime \prime}(\mathrm{x})\right]^{2} \mathrm{e}^{-\mathrm{x}} \mathrm{dx} \leqslant \mathrm{M}_{\mathrm{n}}^{2} \int_{0}^{\infty}[\mathrm{P}(\mathrm{x})]^{2} \mathrm{e}^{-\mathrm{x}} \mathrm{dx}
$$

where

$$
\frac{1}{\mathrm{n}^{4}} \mathrm{M}_{\mathrm{n}}^{2}=\frac{1}{\mathrm{k}_{0}^{4}}-\mathrm{R} \text { and } 0<\mathrm{R} \leqslant \frac{\mathrm{l}}{2 \mathrm{n}}-\frac{1}{6 \mathrm{n}^{2}} .
$$

The polynomials giving equality are unique to within a multiplicative factor.

The eigenfunction corresponding to $\tilde{\lambda}$ actually gives an asymptotic representation for the extremal polynomials. The bounds of Hämmerlin [6] are very convenient for showing this though we shall not carry out these details.

The matrices arising are to be interpreted as discrete Green's functions and their inverses as difference approximations to the boundary value problem found. It is easy to obtain the inverse matrices directly but the degree of approximation is easier to assess for the integral equations. For the Laguerre weights the discrete problem is a boundary difference equation with constant coefficients. The discrete problem in the first derivative case is readily solved and leads to the

Theorem. If $\mathrm{P}(\mathrm{x})$ is a polynomial of degree $\mathrm{n} \geqslant 2$ and $\mathrm{L}_{\mathrm{j}}(\mathrm{x}), \mathrm{j}=1,2, \ldots$, the Laguerre polynomials, then

$$
\int_{0}^{\infty}\left[\mathrm{P}^{\prime}(\mathrm{x})\right]^{2} \mathrm{e}^{-\mathrm{x}} \mathrm{dx} \leqslant \frac{1}{4} \sin ^{-2} \frac{\pi}{2(2 \mathrm{n}+1)} \int_{0}^{\infty}[\mathrm{P}(\mathrm{x})]^{2} \mathrm{e}^{-\mathrm{x}} \mathrm{dx}
$$


with equality holding if and only if $\mathrm{P}(\mathrm{x})$ is a multiple of

$$
\sum_{\mathrm{j}=1}^{\mathrm{n}} \mathrm{L}_{\mathrm{j}}(\mathrm{x}) \sin \frac{\mathrm{j} \pi}{2 \mathrm{n}+\mathrm{l}} \text {. }
$$

This pretty result was given by Turán in [7] and was subsequently rediscovered by the author using the method sketched above.

II. In this part we examine the Legendre case. We shall use the Legendre polynomials $\left\{P_{j}(x)\right\}$ and their normalized forms $\left\{\varphi_{j}(x)=\sqrt{\frac{2 j+1}{2}} P_{j}(x)\right\}$. As before, two integrations by parts and the result $[8$, p. 252]

$$
P_{n}^{(k)}(1)=\left(\begin{array}{c}
n+k \\
n-k
\end{array}\right)(2 k-1) ! !
$$

gives for $i \geqslant j$

$b_{i j}=\sqrt{\frac{2 i+1}{2}} \sqrt{\frac{2 j+1}{2}} \frac{(j-1)(j)(j+1)(j+2)}{48}$

$$
[3 i(i+1)-j(j+1)+6]\left[1+(-1)^{i+j}\right] .
$$

Note that $b_{i j} \geqslant 0$ with equality only when $0 \leqslant i, j \leqslant 1$ or $i$ and $j$ are of opposite parity. This checkerboard pattern shows that taking the basis in the order $\varphi_{0}, \varphi_{2}$, $\varphi_{4}, \ldots, \varphi_{1}, \varphi_{3}, \varphi_{5} \ldots$. transforms $B$ into a similar block diagonal matrix. Along the diagonal are two $1 \times 1$ null matrices consisting of $b_{0,0}$ and $b_{1,1}$ and two positive matrices $E$ and $U$ corresponding respectively to even and odd polynomials. This reflects the facts that the Legendre polynomials of even (odd) order are even (odd) and that even (odd) polynomials go into even (odd) polynomials after differentiating twice.

The characteristic polynomial splits into two factors corresponding to $E$ and $U$. The positive matrices are compared by elements and from the theory of such matrices we conclude that for $n$ odd, the largest eigenvalue of $B$ is the largest eigenvalue of $U$ and for $n$ even, it is the largest eigenvalue of $E$. Thus we are permitted the following qualitative conclusions. If $n$ is even (odd), the extremal polynomial is even (odd). It is unique up to constant multiples and when expressed in terms of the $\varphi_{j}$ may be taken to have positive coefficients except for the coefficients of $\varphi_{0}$ and $\varphi_{1}$ which are zero.

Only the case of even $n=2 m$ will be treated. Odd $n$ lead to exactly the same results. As before, the matrix $E=\left(b_{2 i, 2 j}\right), i, j=1, \ldots ., m$, is a real, symmetric, positive definite matrix. As such it is orthogonally similar to a real diagonal matrix $D=\operatorname{diag}\left\{\lambda_{1}^{-1}\right.$, $\left.\lambda_{2}^{-1}, \ldots, \lambda_{n}^{-1}\right\}$. Here $\lambda_{i}$ are the reciprocals of the eigenvalues. This notation is perhaps confusing here but will be more convenient later. The change of basis carries $\left\{\varphi_{2 i}(x)\right\}$ into $\left\{g_{i}(x)\right\}$. The new basis is orthonormal and is composed of polynomials in even, nonzero, powers of $x$. We have

$\int_{-1}^{1} g_{i} g_{j} d x=\delta_{i j}, \int_{-1}^{1} g_{i}^{\prime \prime} g_{i}^{\prime \prime} d x=\lambda_{i}^{-1} \delta_{i j} \quad i, j=1, \ldots ., m$.
We shall derive a differential equation for the $g_{i}$. For each $i$ define $h_{i}^{\prime \prime}(x)=g_{i}(x), h_{i}^{\prime}(0)=0$, and $h_{i}(1)=0$. Because $h_{i}(x)$ is even, $h_{i}(1)=h_{i}(-1)=0$. Each $g_{i}$ is a linear combination of polynomials orthogonal to 1 so

$$
\int_{-1}^{1} g_{i} d x=0=h_{i}^{\prime}(1)-h_{i}^{\prime}(-1) .
$$

This with the fact that $h_{i}^{\prime}(x)$ is odd shows $h_{i}^{\prime}(1)=h_{i}^{\prime}(-1)$ $=0$.

Let $q(x)$ be an arbitrary even polynomial of degree $n$ without constant term.

$$
q(x)=\sum_{i=1}^{m} c_{i} g_{i}(x)
$$

The orthogonality of the $g_{i}^{\prime \prime}$ shows

$$
\int_{-1}^{1}\left(g_{i}^{\prime \prime} q^{\prime \prime}-\lambda_{i}^{-1} g_{i} q\right) d x=0 \quad i=1, \ldots, m .
$$

Integrating by parts twice and remembering that $h_{i}^{\prime}$ and $h_{i}$ both vanish at \pm 1 shows

$$
\int_{-1}^{1} g_{i} q d x=\int_{-1}^{1} h_{i} q^{\prime \prime} d x
$$

and, using $g_{i}^{\prime \prime}=h_{i}^{(4)}$, the relation $(2.1)$ can be written as

$$
\int_{-1}^{1}\left(h_{i}^{(4)}-\lambda_{i}^{-1} h_{i}\right) q^{\prime \prime} d x=0 .
$$

The expression $\left(h_{i}^{4}-\lambda_{i}^{-1} h_{i}\right)$ is an even polynomial of degree at most $n+2$ and is orthogonal to any even polynomial $q^{\prime \prime}$ of degree at most $n-2$. The expression is naturally orthogonal to all odd polynomials of degree at most $n+1$. The orthogonality conditions require

$$
h_{i}^{(4)}-\lambda_{i}^{-1} h_{i}=d_{i} P_{n+2}+k_{i} P_{n} .
$$

The homogeneous equation can have no polynomial solutions since powers cannot cancel.

We prove $k_{i} \neq 0$ by contradiction. Suppose it were zero. $h_{i}(1)=0$ implies $h_{i}^{(4)}(1)=d_{i}$. The equation has the polynomial solution

$$
h_{i}=-h_{i}^{(4)}(1) \lambda_{i}^{-1} \sum_{l=0}^{\infty} \lambda_{i}^{-l} P_{n+2}^{(4 l)} .
$$

Remembering that

$$
P_{n+2}^{(4 l)}(1) \geqslant 0 \text { with equality only for } 4 l>n+2
$$

and that $\lambda_{i}^{-1}>0$, it is seen that $h_{i}(1)=0$ requires $h_{i}^{(4)}(1)=0$. This is impossible because the polynomial $h_{i}$ cannot be a solution of the homogeneous equation. Define $u_{i}(x)$ by $k_{i} u_{i}=h_{i}$. Using $h_{i}(1)=0, P_{n+2}(1)$ $=P_{n}(1)=1$, we can write $(2.2)$ as

$$
u^{(4)}-\lambda^{-1} u=P_{n}+\left[u^{(4)}(1)-1\right] P_{n+2} .
$$


Let

$$
S_{i}(x)=\sum_{l=0}^{\infty} \lambda_{i}^{l} P_{n}^{(4 l)}(x), \quad T_{i}(x)=\sum_{l=0}^{\infty} \lambda_{i}^{l} P_{n+2}^{(4 l)}(x) .
$$

The solution of $(2.2)$ is

$$
u_{i}(x)=-\lambda_{i} S_{i}(x)+\lambda_{i} \frac{S_{i}(1)}{T_{i}(1)} T_{i}(x) .
$$

The requirement $u_{i}(1)=0$ is manifestly satisfied. The requirement $u_{i}^{\prime}(1)=0$ gives

$$
S_{i}^{\prime}(1) T_{i}(1)=S_{i}(1) T_{i}^{\prime}(1)
$$

which is a polynomial equation for $\lambda_{i}$. In terms of $\lambda$ the equation is

$$
\sum_{l=0}^{\infty} \lambda^{l} \sum_{i+j=l}\left[P_{n+2}^{(4 i+1)}(1) P_{n}^{(4 j)}(1)-P_{n+2}^{(4 j)}(1) P_{n}^{(4 i+1)}(1)\right]=0 .
$$

We now wish to obtain an asymptotic solution of this equation. Let $c(i, j)$ denote the expression in brackets in (2.3). Divide (2.3) by $n$ and let $\eta=\lambda n^{8}$. The resulting equation can be written as $f_{n}(\eta)=0$, where

$$
f_{n}(\eta)=\sum_{l=0}^{\infty} \eta^{l} \sum_{i+j=l} \frac{c(i, j)}{n^{8 l+1}}
$$

We shall use the Hurwitz theorem [9, pp. 156-8] which states that if a sequence of functions $f_{n}$ regular in a domain $D$ converges uniformly to a nonconstant limit function $f$ in $D$, then the zeroes of $f_{n}$ in $D$ tend to those of $f$.

Using the expression for $P_{n}^{(k)}(1)$, it is straightforward to show

$\frac{c(i, j)(8 i+2) ! !(8 j) ! !}{n^{8 l+2}}=\frac{1}{n}(16 i-16 j+4)+\frac{1}{n^{2}} d(i, j)$

where $|d(i, j)|<2^{8 l+4}\left(\begin{array}{c}8 l+2 \\ 4 l+1\end{array}\right)$.

Let us multiply (2.5) by $n$ and substitute in (2.4). With

$$
f(\eta)=\sum_{l=0}^{\infty} \eta^{l} \sum_{i+j=l} \frac{16 i-16 j+4}{(8 i+2) ! !(8 j) ! !}
$$

and

$$
(8 i+2) ! !(8 j) ! ! \geqslant(4 l) ! ! \text { for } i+j=l
$$

we have

$$
\left|f_{n}(\eta)-f(\eta)\right|<\frac{1}{n}\left|\sum_{l=0}^{\infty} \eta^{l} \frac{l 2^{8 l+4}}{(4 l) ! !}\left(\begin{array}{c}
8 l+2 \\
4 l+1
\end{array}\right)\right|
$$

The series appearing in this bound is entire hence is bounded on compact subsets of the plane. The factor $n^{-1}$ assures us that the $f_{n}$ converge uniformly to $f$ on compact subsets of the plane.

Some simple identities show

$$
f(\eta)=2+\sum_{l=1}^{\infty} \eta^{l} \frac{(-1)^{l}}{4^{l}(4 l) !} .
$$

Introduction of the new variable $k$ by $16 k^{4}=\eta$ for positive $\eta$ casts $f$ in the form

$$
f(k)=1+\cos k \cosh k .
$$

The smallest root of $f$ has been designated $k_{0}$. The Hurwitz theorem implies the

TheOREM. If $\mathrm{P}(\mathrm{x})$ is a polynomial of degree $\mathrm{n}$, then

$$
\int_{-1}^{1}\left[\mathrm{P}^{\prime \prime}(\mathrm{x})\right]^{2} \mathrm{dx} \leqslant \mathrm{M}_{\mathrm{n}}^{2} \int_{-1}^{1}[\mathrm{P}(\mathrm{x})]^{2} \mathrm{dx}
$$

where

$$
\frac{\mathrm{M}_{\mathrm{n}}^{2}}{\mathrm{n}^{8}}=\frac{1}{16 \mathrm{k}_{\mathrm{o}}^{4}}+\mathrm{o}(1)
$$

III. Comparison of the asymptotic constants in both cases shows that the true constant is smaller for the second derivative than that given by iterated use of that of the first derivative. For example in the Legendre case the results are $(3.7502082)^{-4}$ and $\pi^{-4}$ respectively. Comparison of the constants is interesting.

\begin{tabular}{l|c|c|c|c}
\hline \hline & $D$ & $D^{2}$ & \multicolumn{2}{|c}{$D^{3}: n=100,125$} \\
\cline { 2 - 5 } Laguerre & $\left(\frac{2}{\pi}\right)^{2}$ & $\left(\frac{1}{k_{0}}\right)^{4}$ & $(0.4472)^{6}$ & $(0.4477)^{6}$ \\
Legendre & $\left(\frac{1}{\pi}\right)^{2}$ & $\left(\frac{1}{2 k_{0}}\right)^{4}$ & $(0.2314)^{6}$ & $(0.2301)^{6}$ \\
\hline
\end{tabular}

One might speculate that the factor of two relation persists for higher derivatives. Some numerical computations are shown for the third derivative for $n$ large enough to give an indication of the asymptotic results. The ratios are respectively 1.93 and 1.95 which are consistent with the factor of two relation. More extensive calculations are planned to substantiate this conjecture. A proof for general $D^{k}$ would appear to be difficult.

\section{References}

[1] E. Schmidt, Über die nebst ihren Ableitungen orthogonalen Polynomensysteme und das zugehörige Extremum, Math. Ann. 119, 165-204 (1944).

[2] J. Todd, Introduction to the Constructive Theory of Functions (Academic Press, Birkhäuser, Basel and Stuttgart, 1963).

[3] E. Kamke, Differentialgleichungen Lösungsmethoden und Lösungen, Akademische Verlagsgesellschaft, Leipzig (1961).

[4] H. Plummer, The numerical solution of a type of equation, Phil. Mag. 32, 505-12 (1941).

[5] H. Weyl, Das asymptotische Verteilungsgesetz der Eigenwerte linearer partieller Differentialgleichungen, Math. Ann. 71, 441-79 (1912).

[6] G. Hämmerlin, Ersatzkernverfahren zur numerischen Behandlung von Integralgleichungen 2. Art. Z.A.M.M. 42, 439-63 (1962).

[7] P. Turán, Remark on a theorem of Erhard Schmidt, Mathematica (Cluj) 2 (25), 2, 373-78 (1960).

[8] G. Sansone, Orthogonal Functions (Interscience, New York, 1959).

[9] J. Gerretsen and G. Sansone, Lectures on the Theory of Functions of a Complex Variable, P. Noordhoff, Groningen (1960).

(Paper 69B3-146) 